\title{
Transcriptome analysis of head kidney in grass carp and discovery of immune-related genes
}

\author{
Jin Chen ${ }^{1,2}$, Cai Li ${ }^{1,2}$, Rong Huang ${ }^{1}$, Fukuan Du ${ }^{1,2}$, Lanjie Liao ${ }^{1}$, Zuoyan Zhu and Yaping Wang ${ }^{1 *}$
}

\begin{abstract}
Background: Grass carp (Ctenopharyngodon idella) is one of the most economically important freshwater fish, but its production is often affected by diseases that cause serious economic losses. To date, no good breeding varieties have been obtained using the oriented cultivation technique. The ability to identify disease resistance genes in grass carp is important to cultivate disease-resistant varieties of grass carp.

Results: In this study, we constructed a non-normalized cDNA library of head kidney in grass carp, and, after clustering and assembly, we obtained 3,027 high-quality unigenes. Solexa sequencing was used to generate sequence tags from the transcriptomes of the head kidney in grass carp before and after grass carp reovirus (GCRV) infection. After processing, we obtained 22,144 tags that were differentially expressed by more than 2-fold between the uninfected and infected groups. 679 of the differentially expressed tags (3.1\%) mapped to 483 of the unigenes (16.0\%). The up-regulated and down-regulated unigenes were annotated using gene ontology terms; 16 were annotated as immune-related and 42 were of unknown function having no matches to any of the sequences in the databases that were used in the similarity searches. Semi-quantitative RT-PCR revealed four unknown unigenes that showed significant responses to the viral infection. Based on domain structure predictions, one of these sequences was found to encode a protein that contained two transmembrane domains and, therefore, may be a transmembrane protein. Here, we proposed that this novel unigene may encode a virus receptor or a protein that mediates the immune signalling pathway at the cell surface.
\end{abstract}

Conclusion: This study enriches the molecular basis data of grass carp and further confirms that, based on fish tissue-specific EST databases, transcriptome analysis is an effective route to discover novel functional genes.

Keywords: Grass carp, Head kidney, cDNA, EST, Immune-related gene

\section{Background}

Grass carp (Ctenopharyngodon idella) is one of the most important freshwater fish, with fast growth, low cost of breeding, and delicious meat. It is widely distributed in China's major river systems. Grass carp is a farmed species that is easily affected by diseases induced by viruses and bacteria; this can cause tremendous economic losses. To date, no excellent breeding varieties have been obtained by the oriented cultivation technique. Because of the long breeding cycle ( $4-5$ years), a hybrid breeding strategy is not feasible. Further, because of the lack of understanding of the genetic background of grass carp, no molecular breeding technology has been applied. The

\footnotetext{
* Correspondence: wangyp.ihb@gmail.com

${ }^{1}$ State Key Laboratory of Freshwater Ecology and Biotechnology, Institute of Hydrobiology, Chinese Academy of Sciences, Wuhan 430072, China

Full list of author information is available at the end of the article
}

discovery of economically important trait-related genes and their functional study may help to establish a molecular breeding technology system in the fish.

ESTs (expressed sequence tags) are partial cDNA sequences obtained after sequencing the ends of random cDNA clones. ESTs were first used in 1991 as an effective new method to discover human genes. Using EST sequences, unknown genomes could be explored at a relatively low cost [1]. With the development of DNA sequencing technology, the cost of sequencing is becoming lower, and the application of large-scale EST sequencing combined with bioinformatics tools for analyzing data is being widely used in different species to find novel genes, for genome annotation, for the identification of gene structure and expression, and in the development of type I molecular markers [2]. In fish, large scale EST sequencing was used in channel catfish

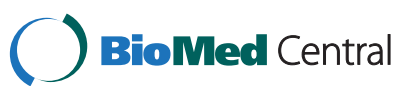


(Ictalurus punctatus) [3], common carp (Cyprinus carpio) [4], and zebrafish (Danio rerio) [5].

In recent years, high-throughput data analysis methods have gradually improved and the genomes of many kinds of fishes have been studied. The fishes that have been studied include zebrafish [6] and fugu [7], as model organisms, and the commercial fishes such as Atlantic salmon [8], sea bass [9,10], rainbow trout [11], Atlantic halibut [12], bluefin tuna [13], turbot [14,15], and Senegal sole fish [16]. In contrast, the molecular biology of grass carp is relatively unknown; currently, there are only 6,915 grass carp ESTs in NCBI's dbEST database. Most functional genomic research on economically important fish is focused mainly on the development of molecular markers, genetic map construction and gene interval mapping, and other basic data accumulation. Research into gene function and its application to breeding is still in the initial stages.

Head kidney is an important immune organ in teleost fish; its role is equivalent to mammalian bone marrow [17]. Head kidney contains a large number of $\mathrm{T}$ and $\mathrm{B}$ lymphocytes, macrophages and granulocytes that are the basis upon which specific and non-specific immunity is acquired.

In this study, we constructed a non-normalized cDNA library for the head kidney of grass carp and obtained 3,027 unigenes including 221 genes of unknown function. We compared the head kidney expression profiles of grass carp infected with grass carp reovirus (GCRV) with normal controls and obtained 22,144 differential expressed tags. Based on a comparison of the differential expressed tags and potential genes with unknown function in the cDNA library, and by identifying gene expression response to GCRV and predicting protein structure, we discovered a novel immune-related gene. This study provides a method for the discovery of novel genes, and reveals the function and the network regulation mechanism of immune-related genes. The results provide a theoretical foundation for molecular design breeding in grass carp.

\section{Methods}

RNA extraction and construction of the CDNA library

Total RNA was extracted from the head kidney of healthy adult grass carp using Trizol reagent (Invitrogen, Carlsbad, CA, USA). The mRNA was isolated using the Oligotex mRNA Kit (QIAGEN, Hilden, Germany). Full length cDNA was synthesized by the Creator ${ }^{\mathrm{TM}}$ SMART $^{\mathrm{TM}}$ cDNA Library Construction Kit (Clontech, CA, USA) following the method described previously [18]. cDNA segments longer than $1 \mathrm{~kb}$ were isolated by electrophoresis, then ligated into pDNR-LIB vector (Clontech) and used to transform competent E. coli DH5 $\alpha$ cells. After growing the colony for 12 hours on an
LB plate containing chloramphenicol, the cDNA library was constructed by selecting mono-clones from the 96well plate. Ethical approval for the work was obtained from Expert Committee of Biomedical Ethics, Institute of Hydrobiology of the Chinese Academy of Sciences. The Reference number obtained was Y12202-1-303.

\section{DNA sequencing and processing of the EST sequences}

10,464 clones were randomly selected from 109 96-well plates. After extracting the recombinant plasmids, 5 ' terminal sequencing was performed using the T7 universal primer (T7: 5' ${ }^{\prime}$-TAATACGACTCACTATAGGG-3'; $\left.\operatorname{Tm}=53.2^{\circ} \mathrm{C}\right)$.

An optimal peak chart was obtained by processing the raw sequence data with basecalling. Next, FASTA format sequences (raw ESTs) were obtained by processing the optimal peak chart using the Phrap program [19] with the Q20 standard. We used crossmatch (Smith and Green, unpublished observations) to remove the pDNRLIB vector sequences and after excluding EST sequences that were less than $100 \mathrm{bp}$ long, we obtained a cleaned EST data set. Clustering of the cleaned ESTs was performed using UIcluster [20]. The UIcluster sequences were assembled using the Phrap program to build a unigene data set for the ESTs from the head kidney of grass carp.

\section{BLAST searches, GO functional classification and KEGG pathway analysis}

We used the NCBI BLAST server [21] to identify sequences that were similar to the sequences in the NCBI nucleotide sequence database $(\mathrm{Nt})$, the protein sequence database $(\mathrm{Nr})$ [22] and the Swissprot database [23] using BLASTN, and BLASTX [24]. Using the EST sequence with the highest homology as a guide, we set the threshold E-value to $\mathrm{E}<1 \mathrm{e}-6$.

We used the BLASTX search results from the Swissprot database and the Blast2GO tool [25] to assign GO functional classification to the unigene sequences. Blast2GO parameters were set as follows: E-Value-Hit-Filter $<1 \mathrm{e}-6$; annotation $\operatorname{cutOff}=55$; other parameters remained at the default values.

KAAS [26] was used to assign the unigene ESTs to pathways based on KEGG Orthology (KO) [27]. Unigenes were mapped to the corresponding KEGG pathways using the comparison method of bi-directional best hit.

\section{GCRV infection of grass carp and preparation of RNA sample}

The GCRV-873 strain was provided by the Gaobo biotechnology company (Wuhan, China). One-year-old grass carp with an average weight of 180-210 g were intraperitoneally injected with $150-200 \mu \mathrm{L}$ GCRV, a 
Table 1 Primers used for semi-quantitative RT-PCR and RACE

\begin{tabular}{|c|c|c|}
\hline Primer & Sequence $\left(5^{\prime}\right.$ to $\left.3^{\prime}\right)$ & Application \\
\hline 291-F1 & ATGTGGGTGATAGTTGGTTTACAAT & Expression study \\
\hline 291-R1 & GTAATITCAGAAGCACAGTTGAGAG & Expression study \\
\hline 357-F1 & CTATCGCATGATTGCCTACTCAGACT & Expression study \\
\hline 357-R1 & ACAACATTITCCATCTCAATCTCAG & Expression study \\
\hline 788-F1 & GGTCTTAACGGAGAGAAGTGCGA & Expression study \\
\hline 788-R1 & GACTCTTCCGGCACGTAACT & Expression study \\
\hline 153-F1 & CCAGCATCACAGTGTTCAGGCAG & Expression study \\
\hline 153-R1 & AGTGTGTAGTTGTGTTCACCCTCC & Expression study \\
\hline$\beta$-actin-F & CAGATCATGTTTGAGACC & Expression study \\
\hline$\beta$-actin-R & ATTGCCAATGGTGATGAC & Expression study \\
\hline 291-F2 & СTCTCAACTGTGCTTCTGAAATTAC & $3^{\prime}$ RACE PCR \\
\hline 291-R2 & ATTGTAAACCAACTATCACCCACAT & 5' RACE PCR \\
\hline 357-F2 & GGTATGATTATGACTAAAGCAGGAC & 3' RACE PCR \\
\hline 357-R2 & GTCCTGCTTTAGTCATAATCATACC & 5' RACE PCR \\
\hline 788-F2 & AGTTACGTGCCGGAAGAGTC & 3' RACE PCR \\
\hline 788-R2 & TCGCACTTCTCTCCGTTAAGAC & 5' RACE PCR \\
\hline $153-F 2$ & GGAGGGTGAACACAACTACACACT & 3' RACE PCR \\
\hline 153-R2 & CTGCCTGAACACTGTGATGCTGG & $5^{\prime}$ RACE PCR \\
\hline
\end{tabular}

dosage of approximately $10^{6} \mathrm{TCID}_{50} \mathrm{~kg}^{-1}$ body weight. The injected grass carp were raised in clean tanks at $28^{\circ}$ C. Three infected grass carp with typical hemorrhage symptoms (infected group, $\mathrm{n}=3$ ) and three uninfected grass carp (healthy control group, $\mathrm{n}=3$ ) were selected at $5 \mathrm{~d}$ after infection for further study. Total RNA was extracted from the head kidney of both groups using Trizol reagent. cDNA was obtained after reverse transcription and used for Solexa sequencing.
Three-month-old grass carp with an average weight of 30-60 g were intraperitoneally injected with $50-80 \mu \mathrm{L}$ GCRV, a dosage of approximately $10^{6} \mathrm{TCID}_{50} \mathrm{~kg}^{-1}$ body weight; fish in the control group were injected with same amount of saline. The grass carp were raised in clean tanks at $28^{\circ} \mathrm{C}$. At $1 \mathrm{~d}, 2 \mathrm{~d}, 3 \mathrm{~d}, 4 \mathrm{~d}, 5 \mathrm{~d}$ after infection ten GCRV-infected carp were selected for further study $(\mathrm{n}=10)$. Ten uninfected fish were selected from the control group at $0 \mathrm{~d}(\mathrm{n}=10)$. The whole fish was immediately used for RNA isolation. cDNA was obtained after reverse transcription and used for the detection of gene expression.

\section{Solexa sequencing and expression profile analysis}

The NlaIII and MmeI digestion method [28] was used to build a 21-bp cDNA tag library of the two groups (oneyear-old), the control group and the GCRV-infected group. The tags in the two libraries end with different Illumina adapter sequences. The raw sequencing read length was $35 \mathrm{bp}$. The Solexa sequencing was performed by the Beijing Genomics Institute (BGI, Shenzhen, China).

The raw sequence data was processed through basecalling, the adapter and low quality sequences were removed, and cleaned 21-bp tags were obtained. We converted the cleaned tag number into the standard (relative) number of transcripts per million (TPM), and calculated the logarithm of TPM for each of the cleaned tags from the control and GCRV-infected groups. We used a dual limit of $\mathrm{P}<0.01$ and FPR (false positive rate) $<0.01$, to find cleaned tags with $\log 2$ Ratio $\geq 1$ or $\log 2$ Ratio $\leq-1$ [29]. The selected tags have differential expression levels of more than 2 -fold in both groups. We then compared the differential expressed tags with the

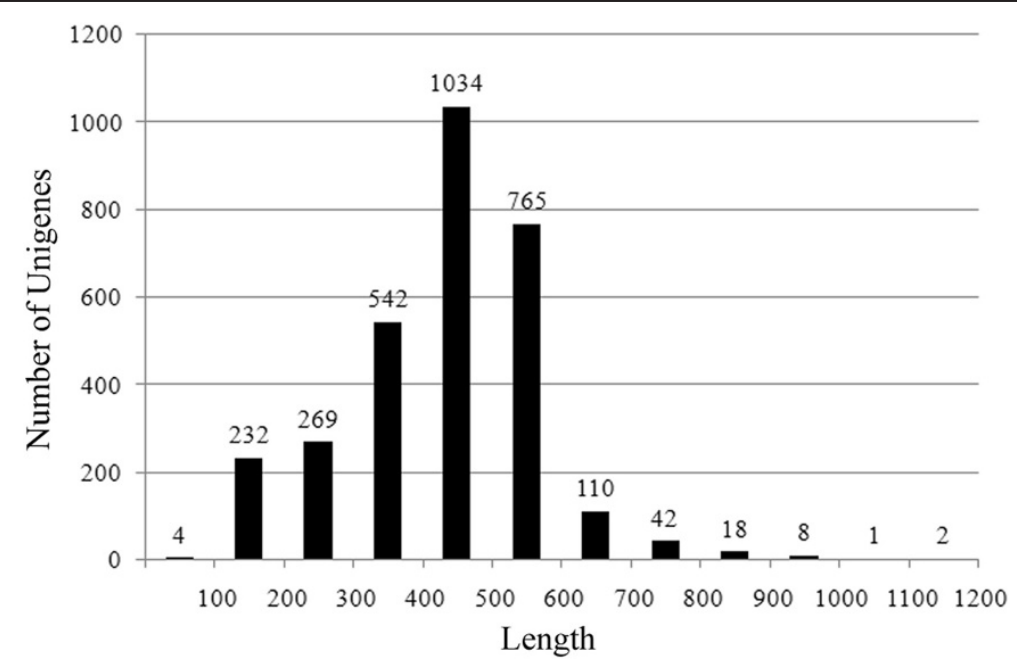

Figure 1 Length distribution of the assembled EST unigenes. The abscissa indicates the length of the unigenes, the ordinate indicates the number of unigenes. 
Table 2 GO functional classification of the unigene data set

\begin{tabular}{|c|c|c|c|}
\hline & GO term & Number of unigenes & $\%$ \\
\hline \multirow[t]{20}{*}{ Biological Process } & cellular process & 899 & 68.0 \\
\hline & metabolic process & 672 & 50.8 \\
\hline & biological regulation & 379 & 28.6 \\
\hline & regulation of biological process & 356 & 26.9 \\
\hline & localization & 250 & 18.9 \\
\hline & establishment of localization & 232 & 17.5 \\
\hline & developmental process & 135 & 10.2 \\
\hline & response to stimulus & 126 & 9.5 \\
\hline & multicellular organismal process & 100 & 7.6 \\
\hline & positive regulation of biological process & 80 & 6.0 \\
\hline & anatomical structure formation & 71 & 5.4 \\
\hline & negative regulation of biological process & 66 & 5.0 \\
\hline & immune system process & 53 & 4.0 \\
\hline & multi-organism process & 22 & 1.7 \\
\hline & growth & 18 & 1.4 \\
\hline & biological adhesion & 14 & 1.1 \\
\hline & locomotion & 14 & 1.1 \\
\hline & reproduction & 14 & 1.1 \\
\hline & reproductive process & 14 & 1.1 \\
\hline & viral reproduction & 4 & 0.3 \\
\hline \multirow[t]{11}{*}{ Cellular Component } & cell part & 1084 & 81.9 \\
\hline & cell & 1084 & 81.9 \\
\hline & organelle & 733 & 55.4 \\
\hline & macromolecular complex & 402 & 30.4 \\
\hline & organelle part & 384 & 29.0 \\
\hline & membrane-enclosed lumen & 140 & 10.6 \\
\hline & envelope & 80 & 6.0 \\
\hline & extracellular region & 32 & 2.4 \\
\hline & extracellular region part & 12 & 0.9 \\
\hline & synapse & 6 & 0.5 \\
\hline & synapse part & 4 & 0.3 \\
\hline \multirow[t]{10}{*}{ Molecular Function } & binding & 770 & 58.2 \\
\hline & catalytic activity & 440 & 33.3 \\
\hline & structural molecule activity & 77 & 5.8 \\
\hline & transporter activity & 70 & 5.3 \\
\hline & transcription regulator activity & 46 & 3.5 \\
\hline & molecular transducer activity & 46 & 3.5 \\
\hline & enzyme regulator activity & 30 & 2.3 \\
\hline & translation regulator activity & 29 & 2.2 \\
\hline & electron carrier activity & 11 & 0.8 \\
\hline & antioxidant activity & 5 & 0.4 \\
\hline
\end{tabular}


Table 3 Unigenes annotated with the GO term immune system process

\begin{tabular}{|c|c|c|c|}
\hline Sequence name & Sequence description & Hit AC & Clustered EST \\
\hline Cluster1088 & fucolectin & Q7SIC1 & 1 \\
\hline Cluster1225 & endoplasmic reticulum aminopeptidase 1 & Q9NZ08 & 1 \\
\hline Cluster1249 & transcription factor sp2 & Q02086 & 1 \\
\hline Cluster1357 & complement c3 & P98093 & 1 \\
\hline Cluster 1410 & b-cell lymphoma 6 protein homolog & P41183 & 1 \\
\hline Cluster1474 & matrix metalloproteinase-9 & P14780 & 1 \\
\hline Cluster 1562 & serine threonine-protein phosphatase subunit & P30153 & 1 \\
\hline Cluster1638 & inosine-5 -monophosphate dehydrogenase 2 & Q3SWY3 & 1 \\
\hline Cluster1667 & chemokine-like factor & Q9UBR5 & 1 \\
\hline Cluster1692 & 60 kda heat shock mitochondrial & Q5ZL72 & 1 \\
\hline Cluster1821 & transcription elongation factor & Q4KLL0 & 1 \\
\hline Cluster1865 & serine threonine-protein kinase tbk1 & Q9WUN2 & 1 \\
\hline Cluster 1872 & dedicator of cytokinesis protein 2 & Q92608 & 1 \\
\hline Cluster1891 & complement c3 & P98093 & 1 \\
\hline Cluster1908 & interferon regulatory factor 4 & Q64287 & 1 \\
\hline Cluster2109 & sh2 domain-containing protein 1a & B2RZ59 & 1 \\
\hline Cluster2173 & bisphosphate phosphodiesterase gamma-2 & Q8ClH5 & 1 \\
\hline Cluster2189 & ig heavy chain v-iii region cam & P01768 & 2 \\
\hline Cluster2214 & complement c3 & P12387 & 2 \\
\hline Cluster2253 & calreticulin & P18418 & 2 \\
\hline Cluster2255 & ap-2 complex subunit sigma-1 & P62744 & 2 \\
\hline Cluster2335 & myosin-if & 000160 & 2 \\
\hline Cluster2337 & adenylate kinase mitochondrial & Q1L8L9 & 2 \\
\hline Cluster2342 & 40s ribosomal protein s14 & P62263 & 2 \\
\hline Cluster2345 & apoptotic chromatin condensation inducer & Q9UKV3 & 2 \\
\hline Cluster244 & MHC I-related gene protein & Q95460 & 1 \\
\hline Cluster2440 & ubiquitin thioesterase otub1 & Q96FW1 & 2 \\
\hline Cluster2466 & nf-kappa-b inhibitor alpha & P25963 & 2 \\
\hline Cluster2474 & toll-interacting protein & A2RUW1 & 2 \\
\hline Cluster2602 & moesin & P26038 & 3 \\
\hline Cluster2612 & beta-2-microglobulin & Q04475 & 3 \\
\hline Cluster265 & myosin-9 & P14105 & 1 \\
\hline Cluster2659 & proteasome maturation protein & Q3SZV5 & 3 \\
\hline Cluster2663 & apoptotic chromatin condensation inducer & Q9UKV3 & 3 \\
\hline Cluster2706 & cd81 antigen & P35762 & 3 \\
\hline Cluster2717 & complement -binding mitochondrial & Q07021 & 4 \\
\hline Cluster2828 & integrin alpha-l & P24063 & 6 \\
\hline Cluster2869 & moesin & P26038 & 8 \\
\hline Cluster2872 & beta-2-microglobulin & O42197 & 8 \\
\hline Cluster2877 & $c-x-c$ chemokine receptor type 4 & P61072 & 8 \\
\hline Cluster2908 & fucolectin & Q7SIC1 & 12 \\
\hline Cluster311 & proteasome subunit beta type-9 & Q8UW64 & 1 \\
\hline Cluster33 & inosine-5 -monophosphate dehydrogenase 1 & P20839 & 1 \\
\hline Cluster490 & paired box protein pax-5 & Q02548 & 1 \\
\hline
\end{tabular}


Table 3 Unigenes annotated with the GO term immune system process (Continued)

\begin{tabular}{llll}
\hline Cluster493 & nucleosome assembly protein 1-like 1-a & Q4U0Y4 & Q9CT8 \\
Cluster588 & cysteine-rich protein 2 & Q96Z06 & 1 \\
Cluster634 & interleukin enhancer-binding factor 2 homolog & P36197 & 1 \\
Cluster668 & zinc finger e-box-binding homeobox 1 & O35904 & 1 \\
Cluster780 & kinase catalytic subunit delta isoform & P35762 & 1 \\
Cluster789 & cd81 antigen & P15314 & 1 \\
Cluster812 & interferon regulatory factor 1 & Q32L31 & 1 \\
Cluster937 & high mobility group protein b3 & Q12904 & 1 \\
Cluster999 & aminoacyl trna synthetase protein &
\end{tabular}

unigenes from the cDNA library using SeqMap [30]; mismatch was set to 0 , and sense and antisense strands were considered in the mapping.

\section{Semi-quantitative RT-PCR and RACE cloning}

Total RNA was used to synthesize the first strand cDNA. Upstream and downstream primers (Table 1) were designed based on the unigene sequences. $\beta$-actin (primers, $\beta$-actin-F and $\beta$-actin-R) was used as the internal reference. PCR and electrophoresis was used to detect the change of expression level.

3' and 5' RACE was performed using the BD SMART RACE cDNA Amplification Kit (Clontech) according to the manufacturer's instructions. Upstream and downstream primers used in the $3^{\prime}$ and $5^{\prime}$ RACE were designed based on the EST sequences (Table 1). Full length cDNA sequences of each gene were assembled using the 3 ' and 5 ' terminal sequences.

\section{Results}

Head kidney cDNA library of grass carp

The storage capacity of the original library was $6 \times 10^{5}$, in the form of the E. coli DH5 $\alpha$ cells that were stored on the 532 96-well plates in a total of 51,072 clones. One hundred randomly selected clones were used for further study. The PCR test results showed that the size of inserts was between 1-3 kilobases, the library

Table 4 Unigenes annotated with the GO term response to virus

\begin{tabular}{llcc}
\hline Sequence name & Sequence description & Hit AC & Clustered EST \\
\hline Cluster2255 & $\begin{array}{l}\text { ap-2 complex subunit } \\
\text { sigma-1 }\end{array}$ & P62744 & 2 \\
Cluster2287 & $\begin{array}{l}\text { interferon-induced } \\
\text { gtp-binding protein }\end{array}$ & Q8JH68 & 2 \\
Cluster2379 & $\begin{array}{l}\text { 40s ribosomal } \\
\text { protein s15a }\end{array}$ & P62244 & 2 \\
Cluster2877 & $\begin{array}{l}\text { c-x-c chemokine } \\
\text { receptor type 4 }\end{array}$ & P61072 & 8 \\
\hline
\end{tabular}

reorganization was $97.85 \%$ and the no-load rate was $2.15 \%$.

\section{EST sequence analysis}

10,464 EST clones were sequenced, and 10,282 FASTA sequences (raw ESTs) with an average read length of $470 \mathrm{bp}$ were obtained. After removing the vector and sequences less than 100 bp long, 7,918 cleaned ESTs (accession no. JK847435-JK855352) were obtained. After clustering and assembly, we obtained 3,027 unigene EST sequences, 802 (26.5\%) of which were contigs and 2,225 (73.5\%) of which were singletons; the library redundancy was $61.78 \%$. Most genes in the library exhibited lowlevel expression, only a small number of genes exhibited high-abundance expression. The number of low expression unigenes, the singletons, was 2,225 (73.5\%); the number of medium expression unigenes, those containing 2-5 ESTs was 641 (21.2\%); and the number of high expression unigenes, those that contained six or more ESTs, was 161 (5.3\%). Only 23 unigenes contained more than 20 ESTs. The average length of the unigenes was $431 \mathrm{bp}$ and $77.33 \%$ of the unigenes were $300-500 \mathrm{bp}$ long (Figure 1).

Table 5 Unigenes annotated with the GO term response to bacterium

\begin{tabular}{lllc}
\hline Sequence name & Sequence description & Hit AC & Clustered EST \\
\hline Cluster12 & histone h2a & P02264 & 1 \\
Cluster1225 & $\begin{array}{l}\text { endoplasmic reticulum } \\
\text { aminopeptidase 1 }\end{array}$ & Q9NZ08 & 1 \\
Cluster1269 & lysozyme c & P85045 & 1 \\
Cluster1910 & akirin-2 & Q25C79 & 1 \\
Cluster2173 & $\begin{array}{l}\text { phosphatidylinositol } \\
\text { phosphodiesterase gamma-2 }\end{array}$ & Q8CIH5 & 1 \\
Cluster2335 & myosin-if & O00160 & 2 \\
Cluster2543 & histone h1 & P06350 & 2 \\
Cluster2861 & histone h1 & P06350 & 7 \\
Cluster566 & histone h1 & P06350 & 1 \\
\hline
\end{tabular}


Table 6 The most represented KEGG pathways in the unigene data set

\begin{tabular}{lll}
\hline Pathway & Mapping genes & Categories \\
\hline Ribosome & 60 & Genetic Information Processing \\
Oxidative phosphorylation & 53 & Metabolism \\
Proteasome & 32 & Genetic Information Processing \\
Spliceosome & 31 & Genetic Information Processing \\
Lysosome & 28 & Cellular Processes \\
Purine metabolism & 25 & Metabolism \\
Endocytosis & 24 & Cellular Processes \\
Regulation of actin cytoskeleton & 24 & Cellular Processes \\
Cell cycle & 19 & Cellular Processes \\
Leukocyte transendothelial migration & 18 & Organismal Systems \\
Pyrimidine metabolism & 17 & Metabolism \\
MAPK signalling pathway & 17 & Environmental Information Processing \\
Antigen processing and presentation & 17 & Organismal Systems \\
Chemokine signalling pathway & 17 & Organismal Systems \\
Tight junction & 16 & Cellular Processes \\
T cell receptor signalling pathway & 16 & Organismal Systems
\end{tabular}

\section{BLAST searches and GO functional classification}

The 3,027 unigenes were used as queries in BLAST searches of the NCBI nucleotide and protein sequence databases and the Swissprot database. 2,713 unigenes (89.6\%) matched sequences in the nucleotide sequence database, 2,162 unigenes (71.4\%) matched sequences in protein sequence database and 1,845 unigenes (61.0\%) matched sequences in the Swissprot database. In all, 2,806 unigenes (92.7\%) matched sequences in at least one of the three databases; the remaining 221 unigenes (7.3\%) were not found (E-value $<1 e-6)$ in any of the three databases and may be novel gene sequences.

Using the gene ontology (GO) classification, we successfully assigned functional annotations to 1,323 of the unigene sequences. In the GO biological process ontology, three terms accounted for the largest proportion of unigenes, they were cellular process, metabolic process and biological regulation; in the GO molecular function ontology, the three most commonly occurring terms were binding, catalytic activity and structural molecule activity; and in the GO cellular component ontology,

Table 7 Mapping genes in fish primary non-specific immune pathways

\begin{tabular}{lcc}
\hline Pathway & Mapping genes & Containing ESTs \\
\hline $\begin{array}{l}\text { Toll-like receptor } \\
\text { signalling pathway }\end{array}$ & 8 & 16 \\
$\begin{array}{l}\text { RIG-I-like receptor } \\
\text { signalling pathway }\end{array}$ & 11 & 20 \\
$\begin{array}{l}\text { NOD-like receptor } \\
\text { signalling pathway }\end{array}$ & 9 & 17 \\
\hline
\end{tabular}

cell, cell part and organelle were the terms that occurred most frequently (Table 2). Of the 1,323 GO-annotated unigenes, 53 were immune system process-related genes (Table 3), 4 were response to virus, and 9 were response to bacterium process-related genes (Tables 4 and 5). Some unigenes were assigned multiple functions. Not all of the unigenes could be mapped to the lower level GO terms.

\section{KEGG pathway analysis}

A total of 989 of the 3,027 were assigned a KEGG ontology (KO) annotation; they were mapped to 201 KEGG pathways. Three most frequently occurring KEGG pathways were ribosome, oxidative phosphorylation, and proteasome. 68 unigenes mapped to immune-related pathways including leukocyte transendothelial migration, antigen processing and presentation, chemokine signalling pathway, and $\mathrm{T}$ cell receptor signalling pathway (Table 6). We found that 28 unigenes from head kidney in grass carp have been reported to be involved in the following pathways, Toll-like receptor signalling pathway, RIG-I-like receptor signalling pathway and the NOD-like receptor signalling pathway (Table 7).

\section{Expression profiling analysis}

By Solexa sequencing, we obtained 7,696,804 and $6,136,889$ raw tags from the transcriptomes of head kidney tissue from grass carp before and after GCRV infection, respectively. After removing low quality sequences, adapter sequences and single copy sequence the cleaned tag numbers were $7,188,005$ and 5,724,526, respectively. 
Table 8 Differentially expressed unigenes annotated as immune-related

\begin{tabular}{llll}
\hline Sequence name & Description & log2Ratio (VP/CP) & Up-Down \\
\hline cichka_Cluster2189.seq. Contig1 & ig heavy chain v-iii region cam & 9.552669098 & Up \\
cichka_Cluster2214.seq. Contig1 & complement c3 & -1.234417227 & Down \\
cichka_Cluster2335.seq. Contig1 & myosin-if & -1.616395009 \\
cichka_Cluster2337.seq. Contig1 & adenylate kinase mitochondrial & -2.622261042 & Down \\
cichka_Cluster2612.seq. Contig1 & beta-2-microglobulin & 14.96510786 \\
cichka_Cluster2717.seq. Contig1 & complement -binding mitochondrial & 2.831849484 & Down \\
cichka_Cluster2828.seq. Contig1 & integrin alpha-l & -3.476196501 \\
cichka_Cluster2872.seq. Contig1 & beta-2-microglobulin & -2.257387843 \\
cichka_Cluster2877.seq. Contig1 & c-x-c chemokine receptor type 4 & -2.941536738 & Up \\
cichka_Cluster2908.seq. Contig1 & fucolectin & -3.57091306 \\
cichka_Cluster2379.seq. Contig1 & 40s ribosomal protein s15a & -2.133495724 & Down \\
cichka_Cluster1269 & lysozyme c & -5.60930435 & Down \\
cichka_Cluster634 & interleukin enhancer-binding factor 2 homolog & -8.383704292 & Down \\
cichka_Cluster812 & interferon regulatory factor 1 & 2.652601218 & Down \\
cichka_Cluster1474 & matrix metalloproteinase-9 & -5.851050959 & Down \\
cichka_Cluster1667 & chemokine-like factor & -8.189824559 & Up \\
\hline
\end{tabular}

The final numbers of non-redundant distinct tags were 152,826 and 105,653 before and after GCRV infection, respectively. All tags were submitted to SRA at NCBI under the accession no. SRA052520.2. Of the distinct tags, 22,144 were differentially expressed by more than 2-fold between the GCRV-infected and uninfected groups.

These 22,144 differentially expressed tags mapped to 3,027 unigenes using SeqMap [30]. Of the differentially expressed tags, 679 (3.1\%) mapped to 483 differentially expressed unigenes $(16.0 \%) ; 145$ of the unigenes were up-regulated genes, 307 were down-regulated genes. The remaining 31 unigenes mapped to tags that exhibited both up and down regulation, and so these unigenes were not included in the statistics. The up- and downregulated genes were mainly annotated with the GO terms, genetic information processing, metabolism, and cellular processes and 16 unigenes were annotated with the GO term immune-related (Table 8). We found 54 tags that mapped onto 42 of the 221 unknown unigenes. These are potentially infection related novel genes; 15 of them were up-regulated between the GCRV-infected and uninfected groups, and 27 were down-regulated genes (Table 9).

\section{Cloning and expression regulation analysis of the novel genes}

Using semi-quantitative RT-PCR, we examined the gene expression changes of the 42 potentially novel unigenes that were detected in the head kidney after viral infection. By comparing the $1,2,3,4$, and 5 day post- infection samples and the samples from the control group, we found four unigenes that showed a significant response to the viral infection: cichka_Cluster153 and cichka_Cluster291 were up-regulated in days 1 and 2 post-infection after which their expressions returned to the starting level; cichka_Cluster357 and cichka_Cluster788 were up-regulated in days 1 and 2 post-infection, and the increased expression levels were maintained till day 5 (Figure 2).

The full-length cDNA sequences of these four unigenes were 2,057 bp (cichka_Cluster291, JQ412736), 2,288 bp (cichka_Cluster357, JQ412737), 1,044 bp (cichka_Cluster788, JQ412738) and 1,387 bp (cichka_Cluster153, JQ412739) encoding polypeptides of 586, 322, 142 and 155 amino acids, respectively. BLAST searches revealed that cichka_Cluster291 can encode a protein that is similar to the vertebrate endonuclease domain containing protein, cichka_Cluster357 can encode a protein that is similar to the vertebrate ankyrin repeat domain 10 protein, cichka_Cluster788 can encode a protein that is similar to the CST complex subunit TEN1; for the cichka_Cluster153 encoded protein, no similar sequences were found in the databases that we searched, suggesting that cichka_Cluster153 may represent a novel gene in grass carp. We used the SMART server [31] to predict the domain structure of the 42 novel unigenes and found that $83.02 \%$ of them contained the endonuclease domain 1 that is found in proteins that are involved in the apoptosis pathway, and $35.22 \%$ contained the ankyrin repeat domain that is present in proteins that are involved in pathways that include the $\mathrm{B}$ cell receptor signalling pathway, the $\mathrm{T}$ cell receptor 
Table 9 Potentially novel differentially expressed unigenes

\begin{tabular}{|c|c|c|}
\hline Sequence name & log2 Ratio(VP/CP) & Up-Down \\
\hline cichka_Cluster1 & -1.30897451703681 & Down \\
\hline cichka_Cluster1004 & -8.18982455888002 & Down \\
\hline cichka_Cluster1074 & -4.68008319087111 & Down \\
\hline cichka_Cluster1080 & 1.5772610962369 & Up \\
\hline cichka_Cluster1095 & 1.70760741456741 & Up \\
\hline cichka_Cluster1139 & -3.01282922395069 & Down \\
\hline cichka_Cluster1321 & -1.17687776208408 & Down \\
\hline cichka_Cluster1418 & 2.57740490960702 & Up \\
\hline cichka_Cluster144 & -2.37056287013824 & Down \\
\hline cichka_Cluster1502 & -2.69938241135805 & Down \\
\hline cichka_Cluster153 & 9.00842862207058 & Up \\
\hline cichka_Cluster155 & -4.35320513151951 & Down \\
\hline cichka_Cluster1567 & -3.23219204494701 & Down \\
\hline cichka_Cluster1689 & -1.24599865006401 & Down \\
\hline cichka_Cluster18 & -8.55458885167764 & Down \\
\hline cichka_Cluster1830 & 5.67155018571725 & Up \\
\hline cichka_Cluster1847 & -1.8154025874359 & Down \\
\hline cichka_Cluster19 & -7.4998458870832 & Down \\
\hline cichka_Cluster1931 & 1.44222232860508 & Up \\
\hline cichka_Cluster2016 & 2.84923580318831 & Up \\
\hline cichka_Cluster2063 & -3.29278174922784 & Down \\
\hline cichka_Cluster219 & 8.44708322620965 & Up \\
\hline cichka_Cluster2432.seq. Contig1 & -1.12271915825313 & Down \\
\hline cichka_Cluster2506.seq. Contig1 & -2.26096007759593 & Down \\
\hline cichka_Cluster2646.seq. Contig1 & -8.18982455888002 & Down \\
\hline cichka_Cluster2651.seq. Contig1 & -8.32192809488736 & Down \\
\hline cichka_Cluster2765.seq. Contig1 & -8.38370429247405 & Down \\
\hline cichka_Cluster291 & 3.07771266869725 & Up \\
\hline cichka_Cluster2966.seq. Contig1 & -5.46317402032312 & Down \\
\hline cichka_Cluster317 & -2.29418310440446 & Down \\
\hline cichka_Cluster357 & 3.48529281620541 & Up \\
\hline cichka_Cluster468 & -1.41853954357293 & Down \\
\hline cichka_Cluster482 & 1.43096228428556 & Up \\
\hline cichka_Cluster559 & 7.82654848729092 & Up \\
\hline cichka_Cluster613 & -1.71345884128158 & Down \\
\hline cichka_Cluster619 & -3.62148837674627 & Down \\
\hline cichka_Cluster625 & 1.46068016483455 & Up \\
\hline cichka_Cluster751 & 1.83711846346595 & Up \\
\hline cichka_Cluster788 & 1.13154390971446 & Up \\
\hline cichka_Cluster790 & -5.47619650111671 & Down \\
\hline cichka_Cluster837 & -2.25442127552909 & Down \\
\hline cichka_Cluster891 & -1.33599920243744 & Down \\
\hline
\end{tabular}

signalling pathway, and the apoptosis pathway. The cichka_Cluster788 unigene contained no obvious structural domains; the cichka_Cluster153 encoded protein contained two transmembrane domains and may be a transmembrane protein.

\section{Discussion}

Currently, there are about 6,915 sequences of grass carp in the public databases. This situation does not reflect the extremely important breeding position of grass carp. In this study, we built a head kidney non-normalized cDNA library of healthy grass carp and obtained 3,027 unigene EST sequences. This library greatly enriches the available genomic data for grass carp and lays an important foundation for the discovery of novel genes and for their functional investigation.

GO analysis revealed that the annotated unigenes were mainly related to genes involved in basic biological processes such as cellular process (25.5\%), metabolic process (19.1\%) and biological regulation (10.8\%). This functional distribution is similar to the EST distributions reported earlier in the head kidney of zebrafish [32] and sea bass [10].

Of the unigenes that were similar to immune-related genes, 66 unigenes were annotated as associated with the immune process; 53 were related to the immune system process, 4 were annotated as response to virus, and

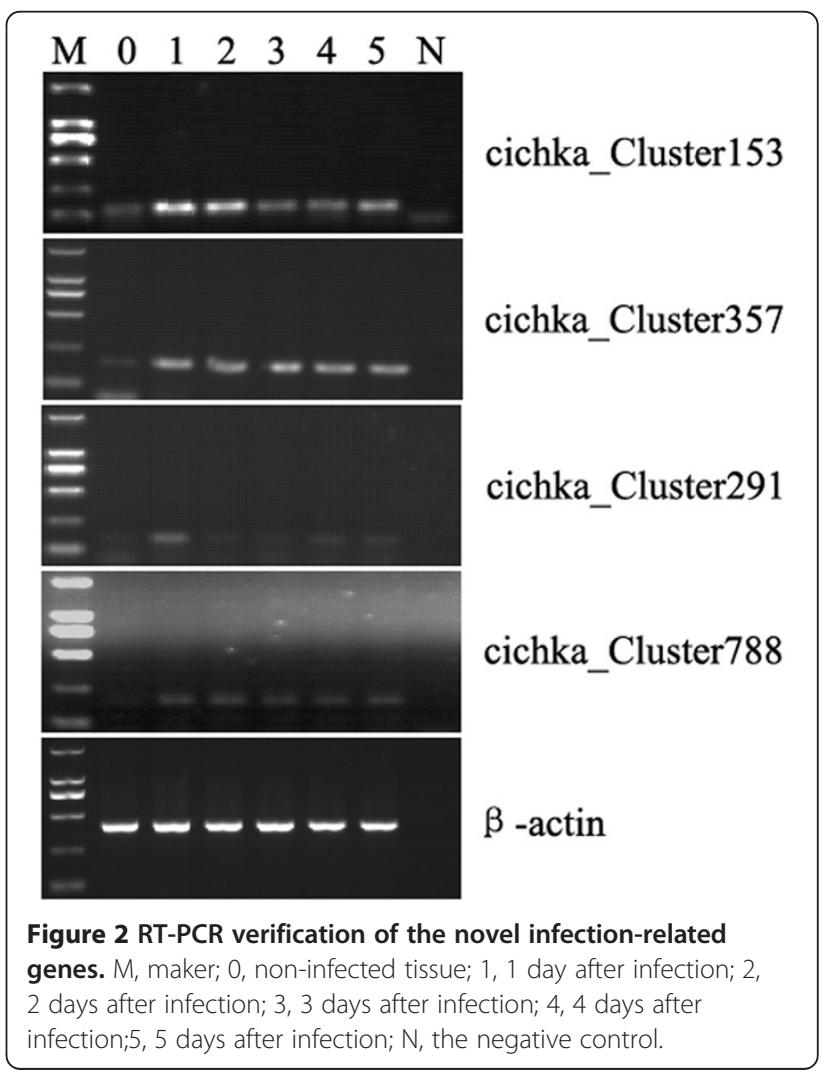




\begin{tabular}{llll}
$\begin{array}{l}\text { Table } \mathbf{1 0} \text { Ten most highly expressed unigenes in the } \\
\text { head kidney of healthy grass carp }\end{array}$ \\
$\begin{array}{llll}\text { Sequence } \\
\text { name }\end{array}$ & $\begin{array}{c}\text { ORF } \\
\text { length }\end{array}$ & Clustered ESTs & \multicolumn{1}{c}{ Description } \\
\hline Cluster2971 & 159 & 1114 & Unknown \\
Cluster2970 & 267 & 251 & hybrid granulin \\
Cluster2969 & 132 & 166 & hypothetical 18 K protein \\
Cluster2968 & 282 & 109 & Unknown \\
Cluster2967 & 273 & 123 & Unknown \\
Cluster2966 & 267 & 78 & hypothetical protein \\
Cluster2965 & 132 & 85 & Unknown \\
Cluster2964 & 0 & 83 & Unknown \\
Cluster2963 & 279 & 63 & Unknown \\
Cluster2962 & 108 & 55 & Unknown \\
\hline
\end{tabular}

9 were related to response to bacteria. Among the 989 unigenes that were assigned $\mathrm{KO}$ annotations, 68 were mapped to immune-related pathways that included leukocyte transendothelial migration, antigen processing and presentation, chemokine signalling pathway and $\mathrm{T}$ cell receptor signalling pathway. By examining the literature, we found that 28 of the unigenes in grass carp head kidney were related to fish genes that were reported to be involved in the Toll-like receptor signalling pathway, the RIG-I-like receptor signalling pathway and the NOD-like receptor signalling pathway. Clearly, head kidney tissue plays an important role in immune processes in fish. EST databases of head kidney tissue are likely to become important resources in which immune-related genes can be identified.

In the 3,027 unigene library of head kidney in grass carp, 7.3\% (221) failed to match any of the sequences in the three public databases that were searched. Of the 10 unigenes that were the most highly expressed in grass carp head kidney, 9 were unknown sequences (Table 10). This could be partly because sequence data for fish is still very scarce, and partly because fish head kidney tissue may contain tissue-specific or species-specific genes. EST databases can be important resources for identifying unknown genes in fish [33-35]. In recent years, the fish transcriptome has been used to study the regulation of gene expression. Pardo et al (2008) conducted a comparative study of turbot expression profiles in the main immune tissue before and after pathogen infection to find genes that were related to immune response and disease resistance [36]. Chini et al (2008) carried out a comparative study of reproductive development-related tissues in bluefin tuna using transcriptome research methods to explore the molecular mechanism of gonadal development and maturity split [13]. Indeed, comparative transcriptome analysis can be used, not only to investigate the mechanisms of expression and regulation of known genes, but also as an effective means to find important and novel function-related genes.

\section{Conclusion}

We carried out a comparative analysis to find differences in the Solexa expression profiles of head kidney in grass carp before and after infection, and identified 42 unigenes of unknown function that showed differential expression in response to the pathogen. After RT-PCR validation of the cDNA and gene structure analysis, we found a potentially novel immune-related gene. Based on its response to viral infection and the prediction that it might encode a membrane protein, we speculate that this novel gene may encode a virus receptor or a protein that mediates the immune signalling pathway at the cell surface. We intend to further investigate the function of this gene in a future study. Our findings confirm that fish tissue-specific EST databases combined with comparative transcriptome analysis are effective tools that can direct the discovery of novel functional genes.

\section{Competing interests}

The authors declare that they have no competing interests.

\section{Authors' contributions}

CJ carried out the experiments and drafted the manuscript. LC and DFK conducted the database searches and bioinformatics analysis. HR and ZZY participated in the study design and in the manuscript preparation. LLJ was involved in the experiments. WYP was overall responsible for the project and finalized the manuscript. All authors read and approved the final manuscript.

\section{Acknowledgements}

The research was financially supported by the Innovation Project of the Chinese Academy of Sciences (KSCX2-EW-N-004-3), the National Key Basic Research Program (2009CB118701), and the Autonomous Project of State Key Laboratory of Freshwater Ecology and Biotechnology (2011FBZ18).

\section{Author details}

${ }^{1}$ State Key Laboratory of Freshwater Ecology and Biotechnology, Institute of Hydrobiology, Chinese Academy of Sciences, Wuhan 430072, China.

${ }^{2}$ Graduate School of Chinese Academy of Sciences, Beijing 100039, China.

Received: 12 February 2012 Accepted: 18 June 2012

Published: 9 July 2012

\section{References}

1. Adams MD, Kelley JM, Gocayne JD, Dubnick M, Polymeropoulos MH, Xiao H, Merril CR, Wu A, Olde B, Moreno RF, Kerlavage AR, Richard Mccombie W, Craig Venter J: Complementary DNA sequencing: expressed sequence tags and human genome project. Science 1991, 252:1651-1656.

2. Nagaraj SH, Gasser RB, Ranganathan S: A hitchhiker's guide to expressed sequence tag (EST) analysis. Brief Bioinform 2007, 8:6-21.

3. Karsi A, Li P, Dunham R, Liu ZJ: Transcriptional activities in the pituitaries of channel catfish before and after induced ovulation by injection of carp pituitary extract as revealed by expressed sequence tag analysis. $J$ Mol Endocrinol 1998, 21:121-129.

4. Savan R, Sakai M: Analysis of expressed sequence tags (EST) obtained from common carp, Cyprinus carpio L., head kidney cells after stimulation by two mitogens, lipopolysaccharide and concanavalin-A Comp Biochem Physiol B Biochem Mol Biol 2002, 131:71-82.

5. Zeng S, Gong Z: Expressed sequence tag analysis of expression profiles of zebrafish testis and ovary. Gene 2002, 294:45-53.

6. Lo J, Lee S, Xu M, Liu F, Ruan H, Eun A, He Y, Ma W, Wang W, Wen Z, Peng J: 15,000 Unique Zebrafish EST Clusters and Their Future Use in 
Microarray for Profiling Gene Expression Patterns During Embryogenesis. Genome Res 2003, 13:455-466.

7. Clark MC, Edwards YJK, Peterson D, Clifton SW, Thompson AJ, Sasaki M, Suzuki Y, Kikuchi K, Watabe S, Kawakami K, Sugano S, Elgar G, Johnson SL: Fugu ESTs: New Resources for Transcription Analysis and Genome Annotation. Genome Res 2003, 13:2747-2753.

8. Adzhubei AA, Vlasova AV, Hagen-Larsen H, Ruden TA, Laerdahl JK, Høyheim B: Annotated Expressed Sequence Tags (ESTs) from pre-smolt Atlantic salmon (Salmo salar) in a searchable data resource. BMC Genomics 2007, 8:209.

9. Chini V, Rimoldi S, Terova G, Saroglia M, Rossi F, Bernardini G, Gornati R: EST-based identification of genes expressed in the liver of adult seabass (Dicentrarchus labrax, L.). Gene 2006, 376:102-106.

10. Sarropoulou E, Sepulcre P, Poisa-Beiro L, Mulero V, Meseguer J, Figueras A, Novoa B, Terzoglou V, Reinhardt R, Magoulas A, Kotoulas G: Profiling of infection specific mRNA transcripts of the European seabass Dicentrarchus labrax. BMC Genomics 2009, 10:157.

11. Govoroun M, Gac FL, Guiguen Y: Generation of a large scale repertoire of Expressed Sequence Tags (ESTs) from normalised rainbow trout CDNA libraries. BMC Genomics 2006, 7:196.

12. Douglas SE, Knickle LC, Kimball J, Reith ME: Comprehensive EST analysis of Atlantic halibut (Hippoglossus hippoglossus), a commercially relevant aquaculture species. BMC Genomics 2007, 8:144

13. Chini V, Cattaneo AG, Rossi F, Bernardini G, Terova G, Saroglia M, Gornati R: Genes expressed in blue fin tuna (Thunnus thynnus) liver and gonads. Gene 2008, 410:207-213.

14. Pardo BG, Fernández C, Millán A, Bouza C, Vázquez-López A, Vera M, Alvarez-Dios JA, Calaza M, Gómez-Tato A, Vázquez M, Cabaleiro S, Magariños B, Lemos ML, Leiro JM, Martínez P: Expressed sequence tags (ESTs) from immune tissues of turbot (Scophthalmus maximus) challenged with pathogens. BMC Vet Res 2008, 4:7.

15. Park KC, Osborne JA, Tsoi SCM, Brown LL, Johnson SC: Expressed sequence tags analysis of Atlantic halibut (Hippoglossus hippoglossus) liver, kidney and spleen tissues following vaccination against Vibrio anguillarum and Aeromonas salmonicida. Fish Shellfish Immunol 2005, 18:393-415.

16. Cerdà J, Mercadé J, Lozano JJ, Manchado M, Tingaud-Sequeira A, Astola A, Infante C, Halm S, Viñas J, Castellana B, Asensio E, Cañavate P, MartínezRodríguez G, Piferrer F, Planas JV, Prat F, Yúfera M, Durany O, Subirada F, Rosell E, Maes T: Genomic resources for a commercial flatfish, the Senegalese sole (Solea senegalensis): EST sequencing, oligo microarray design, and development of the Soleamold bioinformatic platform. BMC Genomics 2008, 9:508.

17. Press CMCL, Evensen O: The morphology of the immune system in teleost fishes. Fish S hellfish Immunol 1999, 9:309-318.

18. Zhu YY, Machleder EM, Chenchik A, Li R, Siebert PD: Reverse transcriptase template switching: a SMART approach for full-length CDNA library construction. Biotechniques 2001, 30:892-897.

19. Ewing B, Hillier L, Wendl MC, Green P: Base-calling of automated sequencer traces using phred. I. Accuracy assessment. Genome Res 1998 8:175-185.

20. Trivedi N, Bishof J, Davis S, Pedretti K, Scheetz TE, Braun TA, Roberts CA, Robinson NL, Sheffield VC, Bento Soares M, Casavant TL: Parallel creation of non-redundant gene indices from partial mRNA transcripts. Future Gen Comp Sys 2002, 18:863-870.

21. : BLAST:: ; http://blast.ncbi.nlm.nih.gov/Blast.cgi.

22. Benson DA, Boguski MS, Lipman DJ, Ostell J, Ouellette BF, Rapp BA, Wheeler DL: GenBank. Nucleic Acids Res 1999, 27:12-17.

23. Boeckmann B, Bairoch A, Apweiler R, Blatter M, Estreicher A, Gasteiger E, Martin MJ, Michoud K, O'Donovan C, Phan I, Pilbout S, Schneider M: The SWISS-PROT protein knowledgebase and its supplement TrEMBL in 2003. Nucleic Acids Res 2003, 31:365-370.

24. Altschul SF, Gish W, Miller W, Myers EW, Lipman DJ: Basic Local Alignment Search Tool. J Mol Biol 1990, 215:403-410

25. Conesa A, Goetz S, Garcia JM, Terol J, Talon M, Robles M: Blast2GO: a universal tool for annotation, visualization and analysis in functional genomics research. Bioinformatics 2005, 21:3674-3676.

26. Moriya Y, Itoh M, Okuda S, Yoshizawa A, Kanehisa M: KAAS: an automatic genome annotation and pathway reconstruction server. Nucleic Acids Res 2007, 35:W182-W185.

27. Kanehisa M, Goto S: KEGG: Kyoto encyclopedia of genes and genomes. Nucleic Acids Res 2000, 28:27-30.
28. Sorana Morrissy A, Morin RD, Delaney A, Zeng T, McDonald H, Jones S, Zhao Y, Hirst M, Marra MA: Next-generation tag sequencing for cancer gene expression profiling. Genome Res 2009, 19:1825-1835.

29. Audic S, Claverie JM: The significance of digital gene expression profiles. Genome Res 1997, 7:986-995.

30. Jiang $\mathrm{H}$, Wong WH: SeqMap: mapping massive amount of oligonucleotides to the genome. Bioinformatics 2008, 24:2395-2396.

31. SMART. http://smart.embl-heidelberg.de/.

32. Song HD, Sun XJ, Deng M, Zhang GW, Zhou Y, Wu XY, Sheng Y, Chen Y, Ruan Z, Jiang CL, Fan HY, Zon LI, Kanki JP, Liu TX, Look AT, Chen Z: Hematopoietic gene expression profile in zebrafish kidney marrow. Proc Natl Acad Sci USA 2004, 101:16240-16245.

33. Yang AF, Zhou ZC, He CB, Hu JJ, Chen Z, Gao XG, Dong Y, Jiang B, Liu WD, Guan $X Y$, Wang $X Y$ : Analysis of expressed sequence tags from body wall, intestine and respiratory tree of sea cucumber (Apostichopus japonicus). Aquaculture 2009, 296:193-199.

34. Chen SL, Xu MY, Hu SN, Li L: Analysis of immune-relevant genes expressed in red sea bream (Chrysophrys major) spleen. Aquaculture 2004, 240:115-130.

35. Cao D, Kocabas A, Ju Z, Karsi A, Li P, Patterson A, Liu Z: Transcriptome of channel catfish (Ictalurus punctatus): initial analysis of genes and expression profiles of the head kidney. Anim Genet 2001, 32:169-188.

36. Pardo GB, Fernández C, Millán A, Bouza C, Vázquez-López A, Vera M, Alvarez-Dios AJ, Calaza M, Gómez-Tato A, Vázquez M, Cabaleiro S, Magariños B, Lemos LM, Leiro MJ, Martínez P: Expressed sequence tags (ESTs) from immune tissues of turbot (Scophthalmus maximus) challenged with pathogens. BMC Vet Res 2008, 4:37.

doi:10.1186/1746-6148-8-108

Cite this article as: Chen et al:: Transcriptome analysis of head kidney in grass carp and discovery of immune-related genes. BMC Veterinary Research 2012 8:108.

\section{Submit your next manuscript to BioMed Central and take full advantage of:}

- Convenient online submission

- Thorough peer review

- No space constraints or color figure charges

- Immediate publication on acceptance

- Inclusion in PubMed, CAS, Scopus and Google Scholar

- Research which is freely available for redistribution 